\title{
Une horticulture maritime ? Pratiques et perceptions de la mer à Tongoa (Vanuatu)
}

A maritime horticulture? Practices and sea perceptions on Tongoa Island

(Vanuatu)

\section{Maëlle Calandra}

\section{(2) OpenEdition}

\section{Journals}

Édition électronique

URL : http://journals.openedition.org/ethnoecologie/3405

DOI : 10.4000/ethnoecologie.3405

ISSN : 2267-2419

Éditeur

Laboratoire Eco-anthropologie et Ethnobiologie

\section{Référence électronique}

Maëlle Calandra, «Une horticulture maritime ? Pratiques et perceptions de la mer à Tongoa (Vanuatu) », Revue d'ethnoécologie [En ligne], 13 | 2018, mis en ligne le 25 juin 2018, consulté le 20 avril 2019. URL : http://journals.openedition.org/ethnoecologie/3405; DOI : 10.4000/ethnoecologie.3405

Ce document a été généré automatiquement le 20 avril 2019.

\section{(†) $\ominus$}

Revue d'ethnoécologie est mis à disposition selon les termes de la licence Creative Commons Attribution - Pas d'Utilisation Commerciale - Pas de Modification 4.0 International. 


\title{
Une horticulture maritime ? Pratiques et perceptions de la mer à Tongoa (Vanuatu)
}

\author{
A maritime horticulture? Practices and sea perceptions on Tongoa Island \\ (Vanuatu)
}

Maëlle Calandra

\section{Introduction}

Les études conduites dans les différentes îles du Pacifique Sud témoignent d'une vision commune de l'environnement marin, lequel est support de territorialisation et s'inscrit dans le prolongement de la terre. Ceci est notamment sensible aux îles Salomon (Hviding 1988, 2006, Johannes 1981, Johannes \& Hviding 2002), en Nouvelle-Calédonie (Leblic 2008, Teulières-Preston 2000), à Tonga (Bataille-Benguigui 1992) ou encore en Polynésie française (Bambridge 2013). À Tongoa, petite île du centre de l'archipel du Vanuatu (Figure 1), la mer est pensée comme un jardin de subsistance et elle est gérée par la hiérarchie locale. Les habitants y possèdent une parcelle "de terre " roara $^{1}$ pour l'agriculture et une parcelle "de mer " natasi où ils œuvrent également pour leur alimentation quotidienne. Qu'ils se rendent dans leurs parcelles de terre pour y retirer de quoi se sustenter, ou qu'ils partent en mer pour y remonter des profondeurs des poissons ou encore qu'ils prélèvent du littoral des crabes et des coquillages, les villageois emploient toujours la même expression : «je pars travailler » (afak posiwosi), signifiant qu'ils s'en vont accomplir un ouvrage relatif à l'alimentation de leur famille. À partir de notre enquête ${ }^{2}$ conduite à Kurumampe (Figure 2), un village situé au nord de l'île, cet article propose de considérer les habitants de Tongoa comme des horticulteurs de la mer et de la terre. Afin d'étayer cette proposition, nous présenterons la territorialisation du littoral et la tenure foncière maritime, les différents savoirs écosystémiques et enfin les principales techniques de prélèvement au sein de cet environnement. 
Figure 1 : L'archipel du Vanuatu

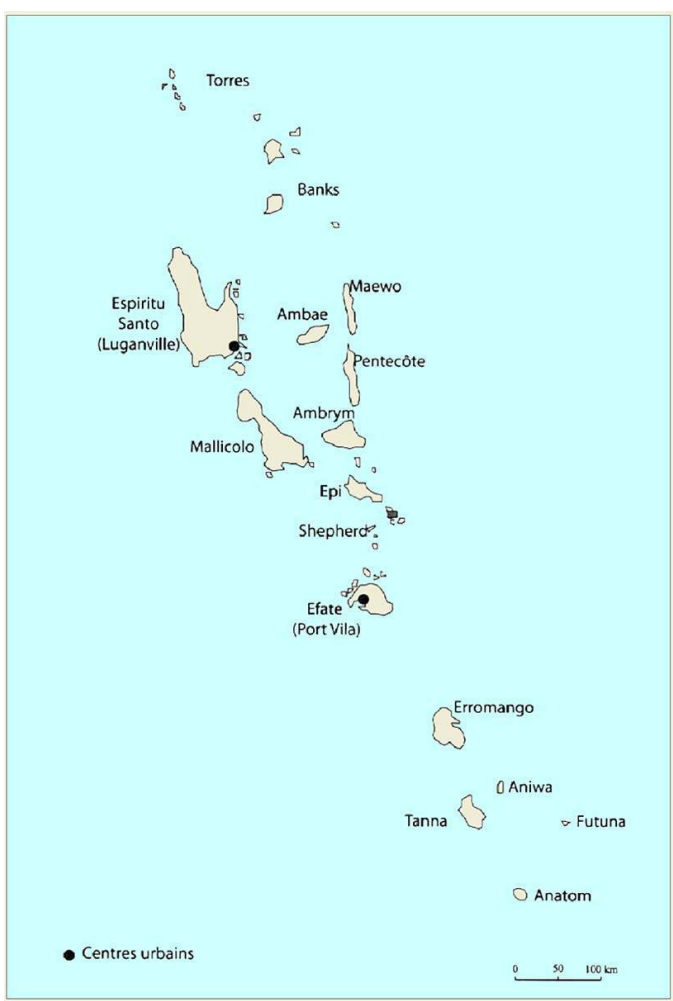

Figure 2 : Le groupement central des lles Shepherd

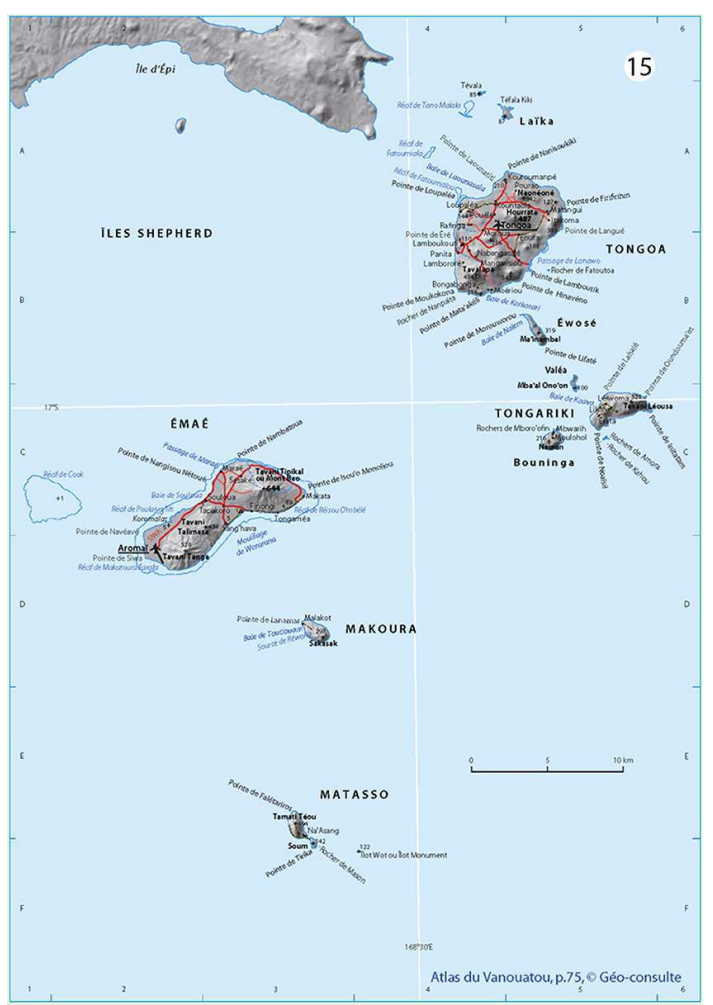

2 L'île de Tongoa est située dans le groupement central des îles Shepherd, au Vanuatu, à proximité du volcan sous-marin nommé Tompuku par les habitants (Calandra 2013). En 
$1452 \pm 1$ an, celui-ci a provoqué un cataclysme d'une telle violence que l'île de Kuwae sur laquelle il se trouvait, jadis la plus grande du pays, s'est scindée en plusieurs îles et îlots (Eissen et al. 1994 : 207, Cole-Dai et al. 1997 : 16 768). D'une superficie de $42 \mathrm{~km}^{2}$, Tongoa est l'un de ces reliquats ${ }^{3}$. Aujourd'hui, les détails morphologiques du paysage témoignent de la violence de l'événement : les falaises morcelées plongent abruptement dans les eaux profondes, les littoraux sont étroits quand ils ne sont pas impraticables, et les mouillages inexistants. C'est du haut des falaises que la houle s'observe et que la hauteur des marées s'apprécie. Il est impossible d'y apercevoir les bas-fonds et les ressources qu'ils abritent, tant l'eau y est profonde. À quelques centaines de mètres du rivage, en direction de l'île d'Epi, c'est le moutonnement des vagues qui attire le regard. Il s'agit de bulles d'hydrogène émanant du volcan. C'est aux abords de cette zone que les pêcheurs viennent jeter leurs lignes de fond car, disent ces derniers, les poissons (naeka) sont attirés par les remous et l'odeur soufrée de l'eau. Les femmes, elles, toujours sur terre et souvent accompagnées de leurs enfants, collectent depuis l'estran des coquillages (finagonda) et des crabes (rakuma). Il n'existe pas de clans de pêcheurs, ou dits " de la mer », comme cela peut être le cas ailleurs dans l'archipel (Bonnemaison 1996). À Tongoa, chacun peut venir prélever des ressources maritimes dans la mesure où aucun interdit (nasikoro) n'est en vigueur.

\section{S’approprier le territoire maritime}

\section{Le partage du littoral}

3 En préambule de ce papier, il convient de livrer quelques informations sur l'organisation sociopolitique de l'île en ce qu'elle détermine la dévolution des droits fonciers et plus généralement l'appartenance à la communauté villageoise. À Kurumampe, les droits d'usage sont régis par une filiation agnatique (la transmission des propriétés foncières se fait en ligne patrilinéaire), chaque individu masculin de chaque famille détient un territoire dans une zone définie, en fonction du groupe matrimonial et politique auquel il appartient. La stratification sociale du village est pyramidale : en haut se trouve le chef du village (nawota lapa), puis ses assistants (nambao ni nawota lapa), et en bas de cette hiérarchie, les gens dits « du commun » (nariei).

4 Le territoire du village est réparti entre dix clans (namatana ni farea). Par clan, nous entendons un groupe de descendance organisé autour de relations directes de consanguinité et possédant plusieurs hectares de terre et bien souvent une parcelle de mer. La résidence est virilocale et chaque clan est placé sous l'autorité d'un nambao ni nawota lapa. Parmi les dix namatana ni farea du village, huit possèdent des segments littoraux différents. Toutefois, suite aux nombreux glissements de terrain engendrés par les séismes et les cyclones de ces dernières années, certains accès conduisant aux parcelles de mer ont disparu. À la fin de l'année 2015, seulement trois parcelles étaient encore accessibles depuis la terre, les autres s'atteignaient seulement par la mer. À cet égard, précisons qu'une parcelle de mer se situe approximativement à une heure de marche du village.

Les habitants racontent que l'appropriation de Tongoa s'est faite cinq ans après l'éruption du volcan (1452 \pm 1 an), suite à l'arrivée progressive des hommes venus en pirogue depuis l'île d'Efate. Une fois débarqués, ils ont marqué les limites de leur territoire depuis le littoral par lequel ils sont arrivés, jusqu'au sommet de l'île où ils se 
sont établis. Tout au long de leur ascension, ils ont signifié leur nouvelle propriété en cassant des branches et en allumant un feu à proximité de leurs habitations. Par ces actes, ils signalaient aux futurs arrivants leur prise de possession d'une terre et délimitaient leurs frontières. Chacune de ces embarcations et chacun de ces groupes d'hommes constitue à présent un namatana ni farea.

Les propriétés maritimes suivent le rivage, une parcelle de mer s'étend d'une extrémité à l'autre des différentes baies de l'île. Il n'existe aucune délimitation matérielle permettant de signifier visuellement les parcelles et d'en marquer les contours, mais plutôt ce que Joël Bonnemaison nomme des «points remarquables» (1981: 254), c'est-à-dire une falaise, un bloc de pierre, un arbre, etc. qui jalonnent les lieux. Les imposants rochers de l'île portent un nom spécifique et bien souvent sont à l'origine d'une histoire coutumière. À titre illustratif, en face du village de Lupalea (Figure 2), se situe en pleine mer un rocher appelé fatu miala (la pierre rouge). Les hommes relatent qu'autrefois celui-ci se trouvait sur l'île, sous un nakafika (Eugenia malaccensis) dont les fleurs de couleur fuchsia, en tombant, lui recouvraient les yeux et le salissaient. Contrarié, le rocher demanda à l'arbre de partir, mais celui-ci refusa, arguant de son antériorité sur les lieux. Le rocher, ne souhaitant pas davantage discuter, partit durant la nuit s'établir en pleine mer, à michemin entre les îles de Tongoa et d'Epi, là où aucun arbre ne pourrait jamais plus le gêner.

Des récits comme celui-ci permettent aux enfants d'assimiler, au fil des narrations, la géomorphologie et les limites du territoire de leurs clans ${ }^{4}$. Les villageois savent ainsi précisément que de tel rocher à tel autre se trouve la propriété maritime du clan de tel village. Rose Mark, une femme de Kurumampe, nous signifia par exemple d'un large geste de la main que depuis le commencement de la baie marqué par un rocher tombé dans l'eau sur lequel pousse aujourd'hui un cocotier, jusqu'au dernier rocher de cette baie, se trouve la mer de son clan, et qu'après celui-ci commence la mer d'un autre clan (Figure 3). Outre les éléments présents dans l'environnement de chacune de ces parcelles, celles-ci sont également distinguées par un nom qui leur est propre. Le système de désignation est fondé sur une observation empirique et articule étroitement le lieu aux événements qui s'y sont déroulés. 
Figure 3 : La parcelle de mer nAmbululua du nAmatanA NI farea NI TANo dont le territoire s'étend jusqu'au rocher au bout de la baie. 5/10/2013, Kurumampe

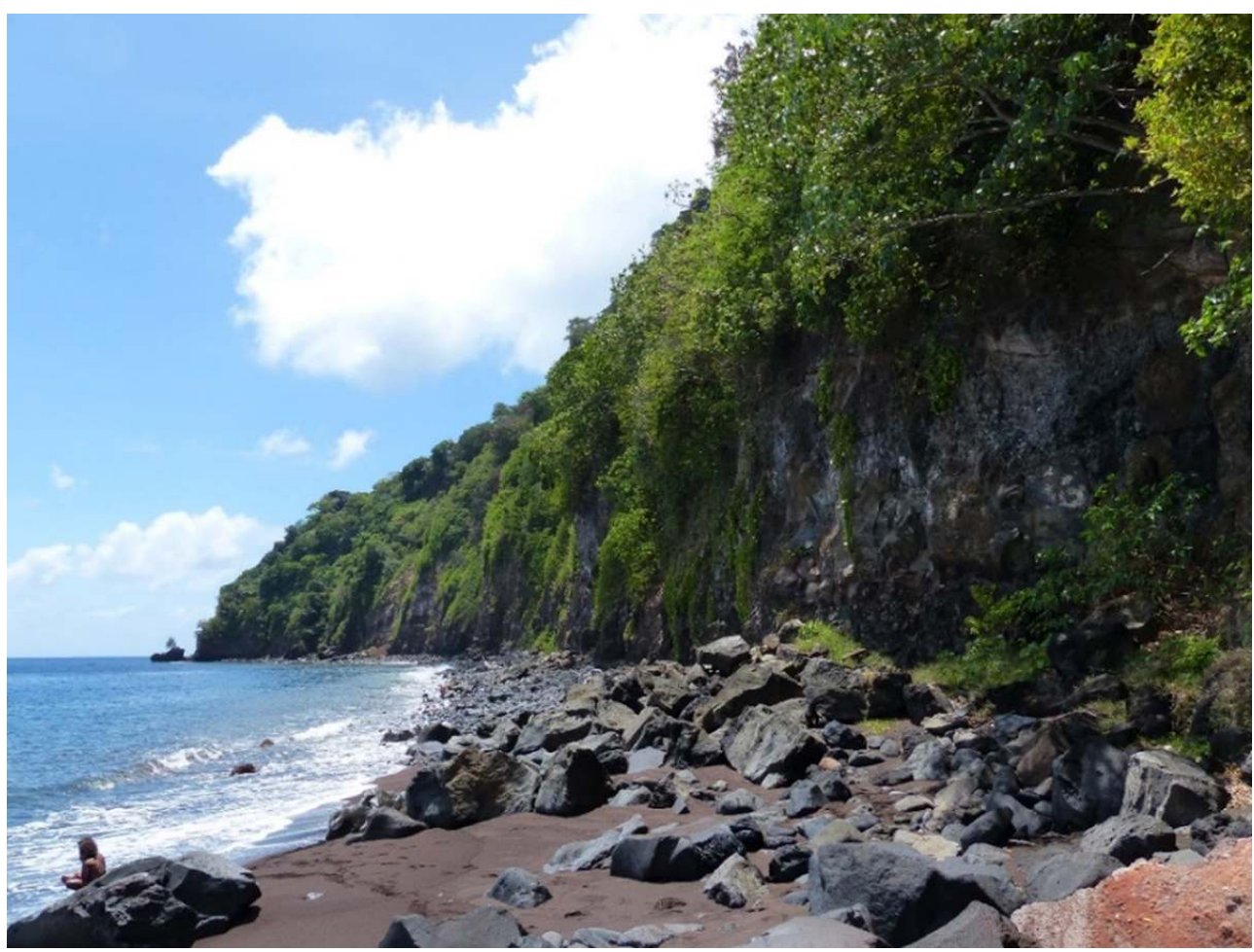

(C) M. CALANDRA

\section{La toponymie maritime}

L'appropriation territoriale s'exprime notamment par la toponymie, car chaque parcelle identifiée est nommée ${ }^{5}$ (comme le récapitule le tableau 1). L'analyse des toponymes révèle une conjonction entre le nom et les particularités écologiques ou géomorphiques de la parcelle. Les toponymes renseignent sur la topographie, à savoir la taille, l'accessibilité ou encore ce qui différencie les «mers » entre elles (ombre, éboulis, danger, etc.). À titre d'exemple, la parcelle maritime du namatana ni farea kiki possède deux noms : " elao ni melu » et «namateana ». Le premier, le plus ancien des deux, évoque l'ombre importante qui se trouve sur son rivage, et le second informe sur le danger de mort que représente à présent celle-ci suite aux nombreux éboulis auxquels elle est exposée. Le nom de la mer du namatana ni farea mataso signifie « la mer où la terre tombe ", car là aussi les glissements de terrain sont récurrents. Après le cyclone Pam survenu le 13 mars 2015, les rochers et les galets situés le long du rivage ont été recouverts d'une épaisse couche de terre, annihilant de surcroît les espèces ichtyologiques qui s'y trouvaient. En dépit de ces spécificités, cette portion de mer reste la plus fréquentée par les villageois, car en plus d'être grande et large, la mer y est toujours plus calme que celle de natoro toro - la parcelle de mer la plus facilement accessible depuis le village, mais où la houle venue du large limite les activités de prélèvement. 
Tableau 1 : Noms et significations des parcelles de mer des différents clans du village de Kurumampe

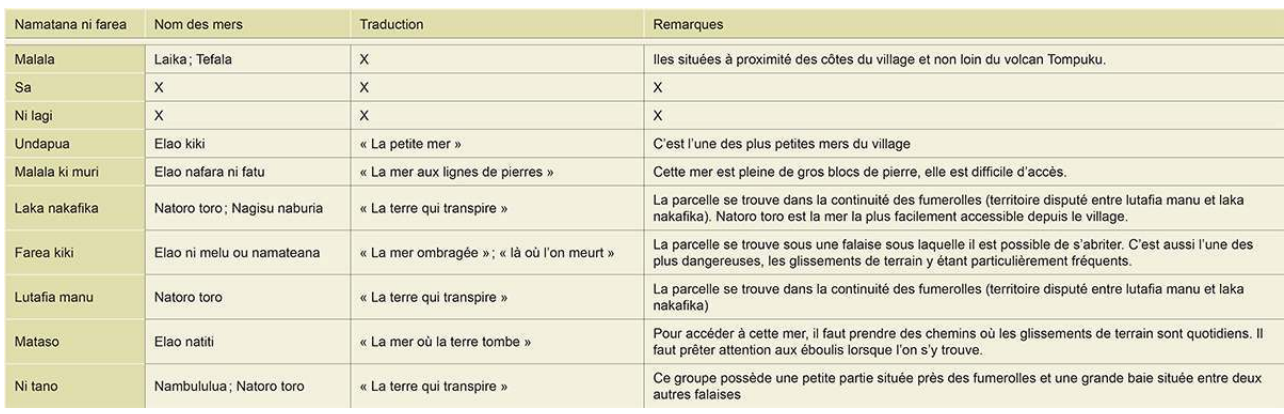

9 De manière similaire à la cartographie terrestre, celle du territoire maritime se transmet par la littérature orale et s'incorpore par l'expérience, au fil des déplacements. Un enfant de 8-10 ans connait le nom des différentes parcelles de mer de son village, il sait où elles se trouvent et il est capable de s'y rendre seul (s'il en a l'autorisation). Ce savoir toponymique, tout comme l'a observé l'anthropologue Hélène Artaud chez les pêcheurs Imrâgen de Mauritanie, a «[...] une fonction autant épistémologique et pratique que sociologique : servant de toile de référents communs aux individus les plus distincts [...] » (2011 : 266). Il révèle également la façon dont la nature est perçue et comprise, mais aussi comment la mémoire des lieux se transmet d'une génération à l'autre.

Toutefois, si les frontières le long du littoral entre chaque parcelle sont facilement identifiables pour les habitants, celles situées dans l'eau et permettant de discerner la mer appropriée (natasi) de la pleine mer (elao), sont bien plus labiles et aléatoires. À la différence du territoire terrestre, en mer existent rarement des " points remarquables " ou des balises permettant de délimiter les territoires entre eux. Ceci est particulièrement remarquable à Tongoa, où il n'existe aucun récif. Si bien que les habitants ne s'accordent pas toujours lorsqu'il s'agit de montrer les limites entre l'intérieur et l'extérieur de leur parcelle. Par exemple, pour Margaret Missel, une femme du namatana ni farea Malala ki muri, le territoire marin d'un clan s'étend du bord de la falaise jusqu'à la première grosse vague dite natafara, à une dizaine de mètres du rivage. D'après Alik Filip, un homme du namatana ni farea Laka nakafika, il se termine là où commence « le très profond », c'està-dire là où l'eau est noire et où personne ne peut atteindre le fond de l'eau. Il se situe ainsi selon lui à une trentaine de mètres de la côte. Pua Daniel, un homme âgé du namatana ni farea Undapua, explique quant à lui que la parcelle se termine là où les pirogues rencontrent la houle et les courants marins venus du grand large, soit à une centaine de mètres du littoral.

11 Au-delà de la limite extérieure de la parcelle, la mer devient " publique » : elle n'est plus appropriée et chacun peut venir y prélever ce qu'il souhaite sans avoir à demander d'autorisation préalable - d'où l'importance de ces limites. Dans cette organisation, le large échappe au contrôle social et à l'ordre hiérarchique auquel les hommes se réfèrent dès lors qu'ils sont sur terre. Le grand large (malo tutmoru ${ }^{6}$ : littéralement, le précipice) est en ce sens considéré par les habitants comme l'équivalent de la brousse forestière ${ }^{7}$ où rien n'est cultivé, où la faune sauvage prospère (en l'occurrence les requins et les raies), mais aussi et surtout où personne ne possède de droits de propriété. Les hommes peuvent s'y rendre librement et s'affranchir de leurs chefs. 
La mer apparait ainsi indissociable de la terre tant elle en constitue un prolongement. Elle est régie par les mêmes codes de propriété foncière que ceux qui régissent la terre. Les territoires maritimes sont structurés par des systèmes de droits d'usage et d'accessibilité. Ils sont placés, comme nous allons le montrer, sous l'autorité du chef de clan qui en contrôle l'accès et y régule les ressources. Cependant, à la différence des parcelles terrestres où les ressources sont individuelles (c'est-à-dire qu'elles appartiennent à une famille nucléaire identifiée), celles de l'espace maritime sont collectives. L'ensemble des membres du clan, voire du village s'ils en demandent l'autorisation au chef concerné, peuvent venir y prélever ce qu'ils souhaitent, dans la mesure où aucune restriction n'est en vigueur.

\section{La tenure maritime}

\section{Les interdits périodiques}

En mer, comme sur le rivage, les espèces présentes sont la propriété des clans et constituent une réserve alimentaire, un «jardin de viande $»^{8}$, pour reprendre le mot d'Alik Philip. Il y a plusieurs générations, les villageois ont mis en place un système de contrôle, afin de pouvoir exploiter durablement les ressources halieutiques 9 . La gestion, l'usage et l'accès de la parcelle reviennent au nambao ni nawota manga. Celui-ci veille à l'équilibre et à la diversité des ressources de la parcelle correspondant au territoire dont il est en charge. Ces appropriations « coutumières » sont reconnues par la Constitution du pays, comme le stipule l'article 73 du chapitre $12:$ "All land in the Republic of Vanuatu belongs to the indigenous custom owners and their descendants ». Leur étendue est précisée par le chapitre 123 de la Loi «Land reform » de 2014 : «'Land' includes improvements thereon or affixed thereto and land under water including land extending to the seaside of any offshore reef but no further $\aleph^{10}$. Depuis les années 1990, le Département des pêches a initié de nombreux programmes encourageant et valorisant ces modes de gestion (Johannes \& Hickey 2004 : 18-19).

Dès lors que les ressources présentes dans la portion de mer d'un chef semblent menacées de disparition, et afin d'enrayer leur épuisement, celui-ci peut décider de poser une mesure de conservation appelée localement nasikoro ${ }^{11}$. La mesure une fois établie, celleci est annoncée par le chef lors des réunions hebdomadaires du village qui se tiennent au farea $^{12}$ et elle est signifiée visuellement par un nasarasara, une structure évoquant une porte ou une croix. Cette structure est mise dans le sol, à l'entrée du chemin conduisant à la parcelle concernée. Sur ce support, l'espèce ou les espèces prohibées sont signifiées par métonymie: des coques vides de coquillages, des carapaces de crabes, des arêtes de poisson, des œufs vidés de mégapodes Megapodius layardi (Figure 4). Généralement, toutes les espèces se trouvant sur une même zone sont interdites, telles que celles de la brousse forestière située à proximité du littoral (mégapodes, crabes), de l'estran (coquillages, crabes) ou de la pleine mer (poissons). Il arrive que la réserve soit intégrale, c'est-à-dire que toutes les espèces et les activités (y compris la baignade et les balades) soient interdites sur l'ensemble de la parcelle. Aussi longtemps que la structure nasarasara est présente, le nasikoro perdure et doit être respecté sous peine de répercussions sociales et de mesures de représailles. 
Figure 4 : Les espèces interdites périodiquement sont signifiées sur ces portails mis à l'entrée du chemin conduisant à la parcelle concernée. Ici, il est annoncé que la collecte d'œufs de mégapodes dits NAMALAO est interdite. Kurumampe, 3/12/2013

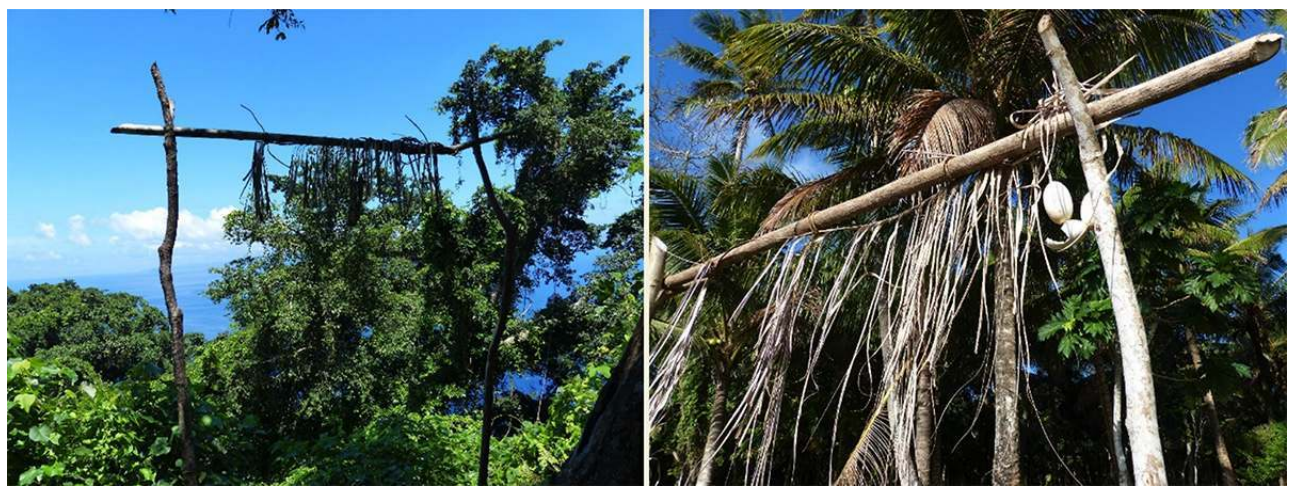

(C) M. CALANDRA

15 C'est aux nate mate, les âmes errantes des défunts de l'île, que revient la responsabilité de l'interdit: ils surveillent et sanctionnent en tuant ou en rendant gravement malade quiconque l'outrepasse. La transgression de cet interdit est localement considérée comme un vol pour lequel une contrepartie doit être versée. Dès lors qu'une personne est prise sur le fait ou alors parce qu'elle associe sa maladie aux nate mate et qu'elle avoue son tort, elle est jugée publiquement au farea. Afin de pouvoir se libérer des nate mate et obtenir le pardon du chef de la mer concernée, la personne fautive doit lui remettre une carcasse de cochon, une natte et de l'argent dont le montant est déterminé par les assistants du chef du village. Les dons sont ensuite redistribués au sein du clan.

Voyons à présent les raisons évoquées par les habitants pour justifier la fermeture temporaire d'une parcelle, et la fonctionnalité imputée à cette mesure. Les entretiens ont permis de distinguer trois motifs principaux, que notre interprétation nous amène à gloser ainsi : économique, stratégique et coutumier. Ces motifs informent à leur tour de la durée de la fermeture, qui va de quelques semaines pour les approches économiques et stratégiques à quelques années pour le cas des fermetures coutumières.

\section{Le motif économique}

Lorsque les populations de poissons et de coquillages semblent en déclin parce qu'un certain nombre de collectes sont infructueuses, le chef de clan (nambao ni nawota manga) auquel la parcelle appartient peut décider d'interdire toute activité de prélèvement. Il est le seul à avoir cette aptitude d'ouverture et de fermeture de la mer. Cette mesure permet de ne pas épuiser les stocks ichtyologiques et prévient les ramassages abusifs. La restriction est maintenue durant quelques mois et peut se prolonger quelques années. La présence de gros spécimens de coquillages sur les parois des rochers déterminera la réouverture de la parcelle par le chef. Le jour où l'interdit est levé, celui-ci retire le nasarasara, demande aux nate mate de ne plus surveiller les ressources et annonce publiquement au farea la réouverture de la parcelle. Dans cette perspective, les hommes apparaissent comme les protecteurs bienveillants de leur environnement. Ce motif de régulation s'inscrit ainsi dans une logique de gestion et de conservation durables des ressources dont les habitants dépendent pour leur subsistance. 


\section{Le motif stratégique}

18 À l'inverse de cette posture conservatrice, les villageois expliquent employer le nasikoro à la manière d'un leurre, pour tromper la méfiance de l'animal. Ce dispositif peut dès lors être considéré comme stratégique et s'il s'apparente au motif économique, il résulte d'observations différentes. Les habitants de l'île imputent aux animaux marins des propriétés analogues à celles que s'attribuent les humains : «ol i kat tingting ! ${{ }^{13}}^{13}$, ils voient, entendent et sont dotés d'une intentionnalité. Leur « intériorité ${ }^{14}$ est similaire à celle des êtres humains, seule leur " physicalité ${ }^{15}$ les en distingue. C'est l'intelligence de l'animal que les pêcheurs cherchent à duper quand ils les chassent. Lorsque les membres d'un clan considèrent que les proies convoitées ne se laissent plus attraper, alors même que ces dernières sont jugées abondantes, la parcelle est fermée ponctuellement de manière à tromper l'animal. Durant ce laps de temps, on suppose que l'animal oublie l'homme et ses ruses, et à la réouverture de la parcelle, il pourra de nouveau être pris sans difficulté.

19 À force de contacts répétés avec les êtres humains, note Nagege Tapao, une femme du namatana ni farea sa, les crabes, les coquillages et les poissons sont capables de les identifier et d'anticiper leurs intentions. Lorsque les coquillages les voient arriver aux abords des rochers, ils se laisseraient tomber dans l'eau où il est plus difficile de les apercevoir, tant ils se confondent par leur couleur avec le sable et les galets. Quant aux crabes, on dit que lorsqu'ils perçoivent la lumière des lampes torches (ils sont principalement collectés la nuit), ils s'enfoncent dans leurs trous pour ne pas se laisser capturer. Enfin, comme le signale Pakoa Abel, un homme du namatana ni farea mataso, les poissons finissent par reconnaître l'hameçon et s'en détournent dès lors qu'ils l'aperçoivent. Par conséquent, explique-t-on, les pêcheurs ne parviennent plus à les piéger.

Dans cette perspective, l'homme n'est pas soucieux de la durabilité des ressources dont il dépend pour son alimentation, mais inquiet de ne plus pouvoir les prélever et apparait comme un prédateur rusé, capable de stratégies pour duper ses proies. À ces deux motifs s'ajoute une mesure non plus économique ou stratégique, mais relevant de la coutume, en ce qu'elle s'inscrit dans le processus de deuil des familles.

\section{Le motif coutumier}

Lorsqu'une personne décède et que son clan possède un segment littoral, une prohibition d'accès est mise en place sur celui-ci pour plusieurs années. La période de restriction est proportionnelle au respect porté à la mémoire du défunt. Si le namatana ni farea ne bénéficie pas d'une façade maritime, il peut demander à un autre clan de fermer sa mer pour lui. En contrepartie, il remettra plusieurs cochons, des tubercules, du kava, des nattes et de l'argent lors de la fermeture puis de la réouverture celle-ci. Ce nasikoro concerne toutes les espèces et toutes les pratiques en lien avec la mer; c'est le plus restrictif de tous, il marque le début et la fin de la période de deuil. Par respect et en souvenir du défunt, explique Makret Josua, une femme du namatana ni farea ni tano, la mer est fermée, car l'âme du mort, trois jours après le décès, descend s'établir en mer. Ce jour correspond à celui où les hommes de la famille sont allés cercler la tombe de pierres, comme le veut le rituel funéraire. C'est au terme de la première période de deuil, soit près de quinze jours après le décès, que l'interdit est instauré. Ce jour-là, le groupe 
accompagné de son chef descend à la mer pour se baigner : depuis le premier jour de deuil, les parents proches du défunt ont cessé de se laver, de se changer de vêtements et de se coiffer. Toutefois, si ceci est la règle énoncée par les villageois, le travail de terrain montre qu'elle est rarement suivie, et révèle même un décalage considérable entre ce qui est dit et ce qui est pratiqué16.

22 L'idée sous-jacente à cette pratique est que l'eau salée permet d'extraire du corps des personnes endeuillées l'odeur du cadavre qu'elles ont veillé, d'où l'intérêt de cette baignade ${ }^{17}$. Une fois que le cortège s'est baigné et que l'eau de mer est comme imprégnée de l'odeur fétide du défunt, celui-ci s'éloigne du rivage. Le chef accroche alors une pièce de vêtement ayant appartenu au mort, à un nasarasara, en forme de croix et planté à la verticale dans le sol. Lorsqu'il dispose l'habit sur la structure (qui évoque celle d'un épouvantail), il murmure au mort de veiller désormais sur les ressources de la parcelle et y noue ensuite deux feuilles de namele (Cycas revoluta) ${ }^{18}$. Cette installation (Figure 5), en plus de signifier l'effectivité de l'interdit, indique que le nate mate d'une personne décédée il y a peu surveille la mer de son clan et par la même occasion fait planer une menace de sanction sur quiconque le transgresserait.

Figure 5 : Suite au décès de sa mère, un NAMBAO NI NAWOTA MANga bloque l'accès à sa parcelle de mer. L'interdit est signifié par les habits de la défunte et deux feuilles de cycas (Cycas revoluta). Kurumampe, 17/08/2015

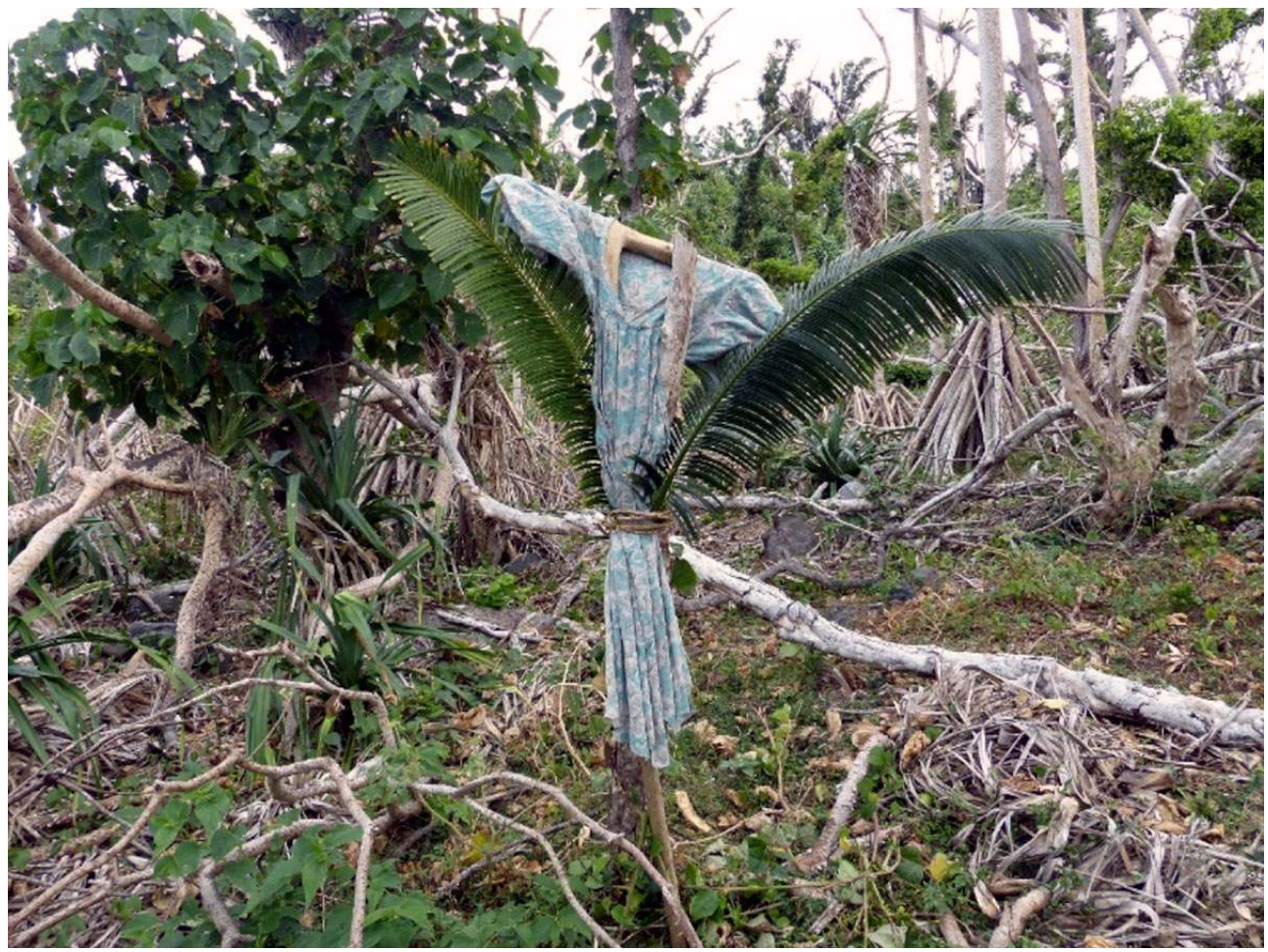

(C) M. CALANDRA

Entre 2012 et 2015 à Kurumampe, une seule «mer » a été fermée à la suite d'un décès. Cette pratique est moins suivie de nos jours, ce nasikoro impliquant de la part de la famille endeuillée de nombreuses dépenses et demeurant actif plus longtemps que les autres interdits. Cependant, ceci n'est pas valable pour toute l'île, puisqu'en 2014, suite au décès du chef d'Euta, l'ensemble des parcelles de mer de ce village ont été fermées pour une durée indéterminée. Il nous faut préciser que ces interdits, s'ils assurent la durabilité 
des ressources, correspondent également à une préoccupation esthétique. Selon les habitants, une parcelle de mer où la diversité ichtyologique est grande est synonyme de «belle mer » (elao wiya). La taille des ressources est également un critère déterminant. Plus les poissons ou les coquillages sont conséquents (lapa ou lalapa), plus la beauté d'une parcelle est appréciée. L'apparence (en l'occurrence la surface de la mer) constitue également un paramètre de cette qualité. Voici ce que dit Makret Josua à ce sujet :

Notre mer [elao nafara ni fatu], c'est la plus belle de Kurumampe ! il y a plein de coquillages et c'est celle où il n'y a jamais de grosses vagues, ce n'est pas comme natoro toro [une autre parcelle de mer du village] où la houle est toujours forte. La nôtre est calme toute l'année. C'est celle où il est le plus facile de ramasser des coquillages, il n'en manque jamais, et tu n'as jamais peur d'être déséquilibré par la force des vagues. 23/10/2014, Kurumampe.

Cette quête du « beau » (wiya) est identique à celle des jardins de terre où l'abondance, la diversité et la grosseur des végétaux sont recherchées. Posséder une «belle mer » emplit de fierté son propriétaire, dont les qualités de gestionnaire seront mesurées à l'aune de l'esthétique de sa parcelle de mer. De la même manière, un chef sera loué par les membres de son clan en fonction de son aptitude à avoir su maitriser les ressources maritimes communes. Pour cette raison certains déploient de nouvelles stratégies de préservation et inventent de nouveaux savoir-faire pour s'assurer la reconnaissance de leurs affins. Le projet mis en œuvre en 2013 par Tipea, un nambao ni nawota manga âgé de 70 ans, fidèle de l'Église adventiste et vivant au village de Lupalea ${ }^{19}$, est à ce titre particulièrement éloquent.

\section{De nouvelles formes de gestion des ressources : nourrir ses poissons}

Un matin de décembre 2013, après avoir constaté qu'il n'y avait plus de gros poissons dans sa parcelle, mais seulement quelques petits spécimens, Tipea conclut que celle-ci ne remplissait plus les critères esthétiques d'une "belle mer». Les habitants de Lupalea allaient principalement pêcher dans sa parcelle, qui n'avait plus fait l'objet de nasikoro depuis une dizaine d'années. De manière à faire face à cette situation, il décida qu'en plus d'apposer un interdit périodique, il viendrait chaque jour nourrir les poissons présents dans sa parcelle afin de les engraisser et de les sédentariser. Tipea expliqua que cette idée lui vint à la suite d'une formation sur l'élevage des poulets organisée par le Ministère de l'Agriculture à Port-Vila, la capitale du pays.

Durant près de dix mois (de décembre à octobre 2013), Tipea déversa dans l'eau de grands paniers emplis de papayes écrasées (issues de ses jardins), de restes alimentaires agrémentés de riz, de thon ou de maquereau en sauce achetés à la boutique du village lorsqu'il en avait les moyens. Il se plaisait à raconter à qui voulait bien l'entendre que sa mer regorgeait de poissons en tout genre, que les coquillages grossissaient à vue d'œil et que bientôt les crabes recouvriraient toutes les pierres du bord de mer. Les poissons restaient dans sa parcelle et l'attendaient chaque matin car, disait-il, ils trouvaient là un environnement favorable à leur établissement ${ }^{20}$.

Cette nouvelle pratique ne manqua pas de susciter l'émoi des habitants de Lupalea, mais également de ceux des villages limitrophes, l'information s'étant répandue sur l'ensemble du territoire par l'intermédiaire du marché. À Kurumampe, l'activité de Tipea ne fit pas l'unanimité et fut même l'objet de vives critiques. Missel Pakoa, un homme du namatana 
ni farea malala ki muri, estimait par exemple que « cela ne se faisait pas » et que seul le nasikoro était la démarche acceptable, car traditionnelle, pour permettre aux espèces de se renouveler. D'autres, comme Elda Roy, également membre de malala ki muri, considéraient qu'il n'était pas d'usage de retenir les poissons en un seul endroit. Les femmes interrogées décrivaient Tipea comme un vieillard sénile, un homme inconscient attirant au bord du rivage des espèces indésirables. En effet, si la plupart des poissons sont consommés, tous ne sont pas bons à retenir en un lieu, à l'image des requins ou des raies, qui sont considérés dangereux pour les pêcheurs. Au demeurant, les femmes l'accusaient de voler les ressources des autres car, disaient-elles, les paniers de nourriture qu'il déversait attiraient les poissons d'autres parcelles vers la sienne.

À l'opposé de ces discours de contestation, certains de nos hôtes souvent plus âgés que les premiers expliquaient que le principe de propriété sur les poissons n'avait pas de sens, dans la mesure où ceux-ci se déplacent librement d'une parcelle à une autre. Pua Daniel, appartenant au namatana ni farea undapua, pensait que la propriété sur les animaux marins ne pouvait s'appliquer qu'aux espèces sédentaires (crabes, coquillages et mégapodes), dans la mesure où celles-ci se trouvent sur terre et évoluent par conséquent sur la propriété d'un clan qu'il est possible d'identifier.

Les savoirs écologiques qui s'expriment au travers de ces pratiques de gestion constituent une autre forme d'appropriation du territoire maritime, car " pour pouvoir gérer, il faut d'abord connaitre » (Roué 2006 : 11). Ces connaissances sont complétées par les activités de prélèvements des hommes et des femmes au sein de cet environnement.

\section{Savoirs et pratiques du milieu maritime}

\section{Des connaissances partagées}

Les activités maritimes nécessitent une connaissance fine du milieu et, comme le remarque Hélène Artaud, exigent " la coïncidence de paramètres naturels spécifiques » (2011: 218). L'auteure qualifie de "sémiologie sensible» cette compréhension de la nature (ibid.). Ce sont ces savoirs qui permettent «à un pêcheur expérimenté de ne rien laisser au hasard et de se concentrer à chaque sortie sur la prise de quelques espèces particulières de poissons » (Hviding 2006: 84). Tout comme pour la plupart des pêcheurs du Pacifique Sud, la lecture de ces indicateurs conditionne le travail et l'accès aux ressources (Di Piazza \& Pearthree $2001:$ 64, Guiot $2003: 4$ ).

31 À Tongoa, selon les phénomènes météorologiques observés, tels que le sens et la force des vents ressentis ${ }^{21}$, la luminosité, le moment de la journée ou encore la hauteur des marées, les hommes et les femmes entreprennent des activités différentes. Afin de pouvoir se laisser dériver le plus longtemps possible et augmenter leurs chances de capturer de gros spécimens, les hommes ont par exemple besoin que la marée soit haute (namuana) lorsqu'ils quittent le rivage. Les femmes, au contraire, opèrent à marée basse (namaati), lorsque le littoral est découvert et vaste. Le moment de la journée est également pris en considération : la pêche est une activité plutôt diurne (en particulier lorsqu'elle est sousmarine), tandis que les collectes se font essentiellement la nuit, quand les coquillages montent au sommet des rochers et que les crabes sortent de leurs trous. Selon les femmes, l'abondance et la disponibilité des ressources qu'elles collectent ne connaissent pas de saisonnalité; elles sont disponibles tout au long de l'année, contrairement à celles concernées par la pêche des hommes. Toutefois, selon les phases lunaires ou la hauteur 
des marées, la qualité de la chair et la quantité des espèces malacologiques varient. Par exemple, il est dit qu'en période de pleine lune, les coquillages sont plus nombreux et que les crabes quittent la brousse forestière, pour venir pondre vers la mer. À l'inverse, les crustacés sont moins visibles lorsque la lune est décroissante.

Lors d'entretiens collectifs avec les femmes, vingt-huit noms d'espèces malacologiques (bivalves et gastéropodes), trois variétés de crustacés et une variété de mollusque (bêche de mer) ont pu être relevés. Il nous faut ici préciser que les espèces ne se trouvant pas sur l'espace intertidal mais dans l'eau sont considérées par les habitants comme des poissons. C'est la niche écologique de l'animal, à savoir la terre, la mer ou l'entre-deux qui détermine sa catégorie générique. À titre d'exemple, la crevette, la langouste ou le poulpe sont des poissons (naeka), dans la mesure où ils évoluent uniquement en pleine mer. En revanche, l'oursin qui se trouve dans l'entre-deux (et non dans les eaux profondes) appartient à la catégorie des coquillages (finagonda).

$\mathrm{Au}$ cours d'entretiens menés avec des hommes de tout âge, cent quarante-trois poissons ont pu être identifiés ${ }^{22}$ (raies et requins compris); tous sont nommés par un terme spécifique en langue vernaculaire. Les espèces pêchées sont réparties, selon la taxinomie locale, en soixante-quatre familles différentes, elles-mêmes rattachées à vingt-huit genres différents. Encore partagés par le plus grand nombre, ces savoirs relatifs à la faune ichtyologique sont répartis de manière plutôt égale entre les sexes. Cependant, certains sous-domaines de connaissances plus spécifiques, comme la pêche, sont l'apanage des hommes, tandis que la collecte des coquillages reste celui des femmes.

En nakanamanga, il n'existe pas de terme générique couvrant l'ensemble des pratiques halieutiques, mais une expression singulière pour chacune des techniques de pêche qui vont à présent être présentées. À la différence des travaux accomplis dans les jardins de terre, le travail dédié à l'espace maritime répond à une logique de division plus stricte entre les tâches masculines et les tâches féminines.

\section{Une appropriation genrée des ressources maritimes}

Le territoire maritime se décompose en quatre espaces de travail : la brousse située à proximité du littoral, l'estran (euta), l'intérieur des parcelles de mer (elao) et l'extérieur des parcelles de mer, c'est-à-dire la haute mer (malo tutumoru). L'occupation de ces zones par les habitants varie selon les sexes et engendre de surcroît des techniques et des dynamiques de travail différentes. La terre est un écotype plutôt féminin, tandis que la mer est plutôt un domaine d'intervention masculin. Néanmoins, il n'existe pas d'interdit spécifique pour chacun des deux sexes. Cette segmentation du territoire se fonde sur des différences de capacités idiosyncrasiques attribuées localement à chacun des deux sexes. Les femmes ne s'aventurent pas en mer, car elles expliquent ne pas connaître la navigation, mais aussi ne pas avoir la force physique pour faire face à l'impétuosité des flots et diriger une pirogue sans vaciller. Cependant, à Panita (le seul village situé au niveau de la mer à Tongoa) quelques femmes savent manier la pagaie. Il arrive aussi que des femmes accompagnent leur époux partant pêcher à bord d'un bateau en aluminium à moteur hors-bord ${ }^{23}$. Il est également possible pour les hommes de participer au travail de collecte le long du littoral, notamment la nuit lorsque leur épouse part capturer des crabes.

Les activités de ramassage concernent ainsi plutôt les femmes, tandis que les activités impliquant une lutte ou l'emploi d'une certaine force physique relèvent davantage du 
domaine des hommes. Toutefois, les activités pratiquées à l'orée de la forêt ou en contrebas des falaises ne sont pas genrées. Dans cette zone, les habitants chassent les pétrels juvéniles (Pterodroma occulta) ${ }^{24}$ et collectent les œufs de mégapodes, deux espèces endémiques au pays, mais menacées de disparition (Dekker et al. 2000). Les pétrels juvéniles sont chassés sur les îles de Laeka et Tefala situées en face du village de Kurumampe. Tous les ans, entre les mois de mars et avril, les juvéniles qui ne savent pas encore voler et qui nichent sur le sol sont collectés en grande quantité, selon le quota imposé par le chef du village ${ }^{25}$. Les chasseurs partent durant plusieurs jours à Laeka où ils remplissent de grands sacs de ces oiseaux marins (préalablement plumés, vidés et roussis sur le feu) et rentrent au village terminer de les cuire sur des pierres chaudes pour ensuite les vendre ${ }^{26}$.

Bien que les activités maritimes puissent être pratiquées à plusieurs, elles n'en restent pas moins individuelles. Chacun travaille pour sa famille nucléaire et les prises sont rarement partagées. Il n'existe pas de forme de pêche collective nécessitant la force de l'ensemble des membres de la communauté, comme cela peut être le cas au nord de l'archipel, notamment aux îles Banks (Vienne 1984 : 165). Les rares situations induisant une collaboration impliquent qu'un très gros poisson, tel un requin, ait mordu au dispositif et que la force d'un seul homme ne soit pas suffisante pour le sortir de l'eau. La prise est alors la propriété de la personne ayant placé l'hameçon et qui décidera de la partager ou non. Par ailleurs, dans la mesure où les ressources maritimes ne font l'objet d'aucune conservation, elles ne sont pas collectées en grandes quantités. Les habitants se rendent en mer en fonction de leurs besoins et prélèvent de quoi se nourrir pour un ou deux repas. Il est notable que les activités entreprises par les femmes, bien que moins productives que celles des hommes, soient plus régulières, plus fiables en termes de récolte et plus facilement accessibles. En effet, lorsqu'elles se rendent en bord de mer, elles sont toujours certaines de rapporter quelque chose. À l'inverse, la pêche des hommes est plus incertaine et plus variable tant elle est dépendante des saisons et de la météorologie.

\section{Les collectes des femmes}

Venues en groupe et souvent issues d'un même clan, les femmes œuvrent depuis la frange littorale. Couteau à la main, elles arpentent le bord de mer pour y récolter des coquillages et des crustacés en partant d'un point $A$ vers un point $B$, pendant en moyenne cinq heures. Chacune part d'un endroit différent, voire opposé (elles se répartissent spontanément les extrémités), pour ne pas risquer de soulever les mêmes cailloux, puis au fil de leur avancée, elles se rejoignent progressivement. La collecte est terminée lorsque l'ensemble de la baie a été exploré.

Le travail implique une certaine endurance physique: le corps à moitié immergé dans l'eau, il faut parvenir à garder l'équilibre malgré la force des vagues, tout en veillant à la chute des cailloux déplacés. Les femmes passent leur main dans les trous, les interstices, sous les rochers, et retournent chacune des pierres qui semble abriter les bivalves et les gastéropodes désirés. Elles développent de fines capacités visuelles et tactiles qu'elles perfectionnent tout au long de leur engagement avec leur espace de travail. Sans voir, mais au toucher, selon le relief et la forme de la coquille, leurs mains entraînées détectent et reconnaissent les coquillages comestibles ${ }^{27}$. Pendant qu'une main palpe le rocher et cherche à découvrir un coquillage, la seconde tient le couteau et se cramponne au rocher 
(Figure 6). Lors de leurs collectes, les femmes disent ne pas chercher la diversité mais la quantité, ainsi que les coquillages qu'elles aiment le plus manger.

Figure 6 : Alison Remon en train de repérer, avec sa main, les coquillages à ramasser sous les rochers. Elle les décollera de la paroi à l'aide de son couteau pour les mettre dans son tissu afin que les vagues ne les emportent et pour que ses mains restent libres. Kurumampe, 26/09/2014

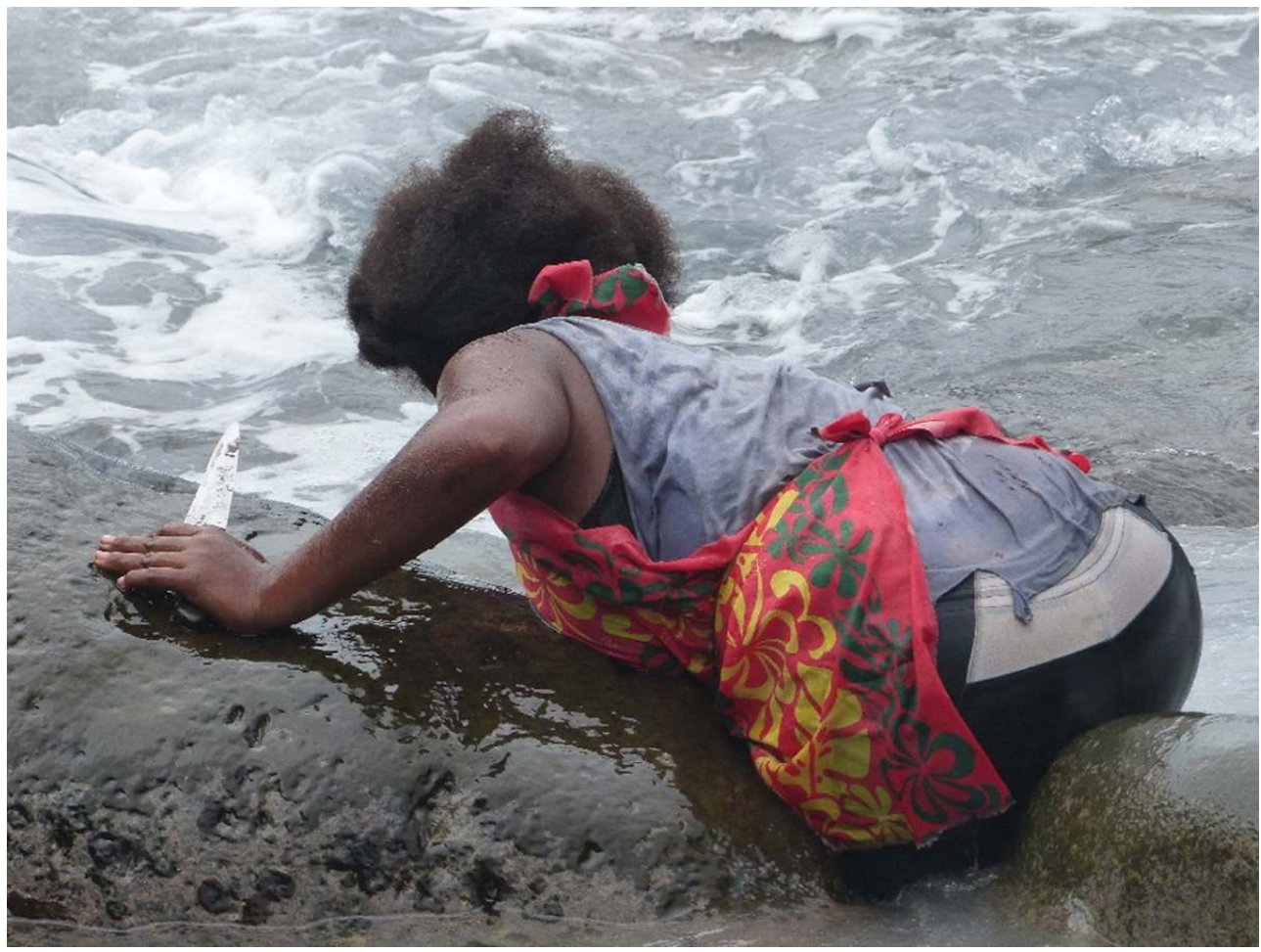

(C) M. CALANDRA

40 Les connaissances que les femmes ont de cet écosystème leur permettent de faire des associations malacologiques et ainsi de gagner en efficacité durant leur ouvrage. Elles savent par exemple que telle taille de rocher ou que telle quantité d'eau signale qu'un coquillage en particulier peut s'y trouver. Par ailleurs, plus la marée est basse, plus les coquillages sont discernables. Depuis leur enfance, elles ont été familiarisées à cet environnement ; les enfants, dès leur plus jeune âge, accompagnent les femmes de leur clan lors de leurs expéditions et apprennent à nager à ces occasions. Elles leur indiquent où trouver les coquillages et nomment les espèces trouvées. Pendant que les mères collectent, les plus jeunes les imitent et se font corriger quand leurs gestes manquent de précision, aiguisant ainsi leur éducation sensorielle. Nous nous souvenons, par exemple, de Sonia demandant à sa mère, Nagege Tapao, où trouver des oursins pour les faire rôtir sur le feu avec ses compagnons de jeu. Celle-ci lui indiqua un rocher et lui expliqua comment les sortir de l'eau sans se blesser avec les aiguilles de l'échinoderme. L'incorporation de ces savoirs par un affinement des capacités en contexte d'application a été qualifiée par l'anthropologue Tim Ingold d'« éducation de l'attention» (Ingold 2000 : 167) ${ }^{28}$. À Tongoa l'enfant n'apprend pas à devenir un bon travailleur à l'école, mais avec ses parents et en situation (dans les jardins ou en bord de mer). 


\section{La pêche des hommes}

41 Si les poissons sont consommés toute l'année, c'est surtout durant la saison chaude (de novembre à février) que les hommes s'adonnent à leur capture, lorsqu'il y a moins de travail dans les jardins ${ }^{29}$. Ce moment correspond à la période durant laquelle la mer est vue comme " généreuse » et "grasse », car les vives eaux sont plus fréquentes : elle est alors dite elao matolu (l'adjectif "matolu» est également employé pour décrire la corpulence d'un animal ou d'un être humain). Lorsque la mer est décrite en ces termes, c'est qu'elle monte haut et délivre de gros poissons, notamment des espèces pélagiques (vivaneaux, thons, bonites, etc.). Ces observations s'opposent à celles de la saison «froide» lors de laquelle la mer est perçue comme «maigre »(elao e mangura): les marées montent moins haut et fournissent seulement de petits poissons. La pêche peut se faire depuis la côte, en pleine mer ou sous l'eau (Figure 7).

Figure 7 : Deux hommes, chacun dans sa pirogue à balancier, en train de partir pêcher vers le large. Kurumampe, 21/08/2015

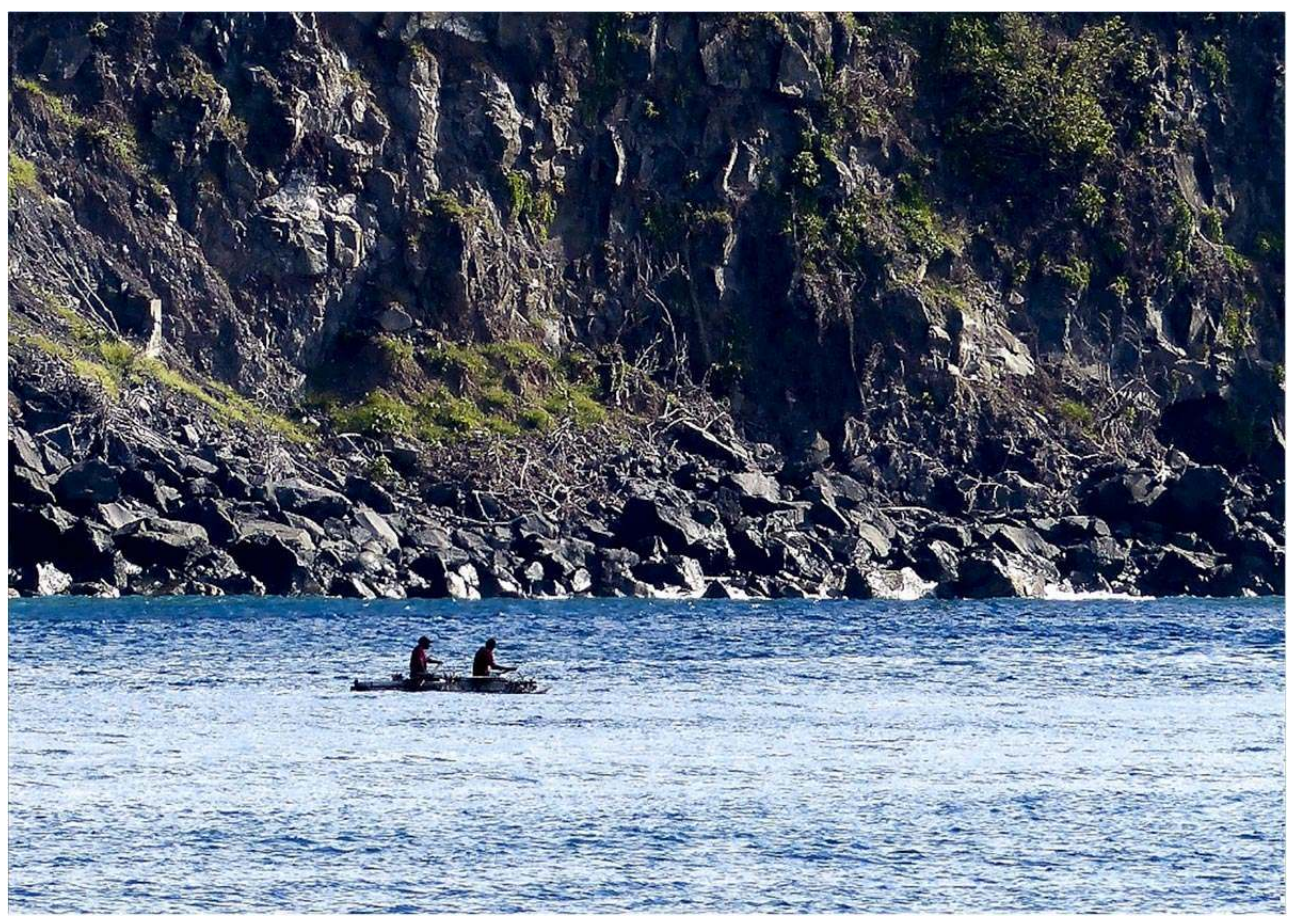

(C) M. CALANDRA

La pêche est considérée comme une activité aléatoire : le pêcheur ${ }^{30}$ ne sait jamais s'il parviendra à attraper des poissons, mais aussi s'il sera épargné par la force des vagues. Face à cette variabilité, et de manière à s'assurer de bonnes prises, un ensemble de conditions particulières doit être respecté - dont la plus importante est de garder l'entreprise secrète. Un homme qui part en mer ne dévoile pas son intention, mais prétexte un travail dans ses essarts. Son épouse est elle aussi tenue à la discrétion, et si quelqu'un réclame son mari, elle dira qu'il est parti œuvrer dans leurs jardins. Tenir son projet confidentiel constitue l'une des préconditions symboliques de la pêche car les poissons, comme nous l'avons déjà souligné, pourraient bien deviner les intentions du pêcheur et ainsi ne pas mordre à l'hameçon. Par ailleurs, les hommes racontent que leur 
discrétion les protège de la jalousie de pêcheurs malintentionnés qui pourraient demander à leur nate mate de détourner les poissons des dispositifs de pêche. Ainsi, pour ne pas éveiller les soupçons, le pêcheur descend à la mer en empruntant des accès dissimulés qui traversent la forêt, lieu où il cache et récupère sa pagaie (nawose) avant et après chaque pêche.

43 Afin de s'assurer de bons résultats et de gagner en prestige en étant reconnus comme de bons pêcheurs par la communauté villageoise, les hommes confectionnent des appâts différents dont ils gardent les techniques de fabrication secrètes. Ils les élaborent à partir de viscères de vache ou de cochon lorsqu'ils souhaitent pêcher les requins ou y accrochent des morceaux de poulpe pour capturer des poissons de moins grosse taille. Ils fabriquent également un leurre à partir d'une plante appelée nawota nano (Crinum asiaticum), qu'ils cultivent spécifiquement à cet effet dans leurs jardins. Celle-ci est appréciée pour sa chair translucide à laquelle ils donnent une forme de poisson ou de petit poulpe et qu'ils s'emploient à déplacer dans l'eau afin de reproduire leurs mouvements. Les clapotis et la brillance du leurre éveillent la curiosité des caranges et des bonites qui viennent alors mordre au dispositif. D'autres utilisent des feuilles, des pierres ou même des prières pour charmer les poissons. Plus les prises sont conséquentes, plus la reconnaissance sociale du pêcheur grandit.

44 L'ensemble de ces techniques de prélèvement est complété par l'engagement des corps. Les hommes cherchent à se confondre avec l'environnement dans lequel ils évoluent pour ne pas être vus des poissons et s'assurer de bonnes prises. En nakanamanga, pêcher se dit palako, ce qui signifie littéralement « surveiller ». Faute de voir ou de sentir leurs proies depuis leurs embarcations, et afin de parvenir à leurrer l'animal appété, les pêcheurs développent une capacité à se mouvoir et à faire de leur corps le prolongement de leur outillage. Ils cherchent à se confondre avec l'environnement dans lequel ils se meuvent pour ne pas être vus des poissons, tout en les attirant avec leurs appâts. Une fois le dispositif mis à l'eau, ils s'appliquent à ne faire aucun bruit pour ne pas effrayer les proies potentielles et ne pas risquer qu'elles s'échappent. Ils sont attentifs au moindre tressautement de leur fil, qu'ils retiennent entre l'index et le pouce; dès qu'il se tend et commence à frémir, c'est d'un coup sec et précis que l'animal est ferré. Il est ensuite remonté à la surface de l'eau avec agilité et précaution afin qu'il ne se décroche pas, puis il est tué pour éviter que les sursauts de son agonie fassent résonner la pirogue sous l'eau.

\section{Conclusion}

Cet article a proposé une description ethnographique précise des différentes logiques d'appréhension du milieu maritime, des processus de domestication de la mer, des connaissances écologiques qu'elles mobilisent et des techniques de prélèvement développées localement, afin d'approfondir une réflexion sur les conditions d'appropriation de cet espace. Omniprésente par les activités qu'elle autorise et nécessite à la fois, ainsi que par la subsistance qu'elle assure, la mer est sous l'emprise de l'action humaine. S'il apparaît pour le géographe Joël Bonnemaison que

« l'originalité des îles mélanésiennes est d'avoir formalisé jusqu'à l'extrême cette séparation écologique [entre la terre et la mer] et d'avoir fondé sur elle un système géographique de différenciation » $(1996: 226)$, encouragent à penser l'insularité comme un territoire non pas clos, mais ouvert. La 
rupture entre la terre et les flots n'est pas nette, tant ils semblent s'emboîter. Les perceptions dont la mer fait l'objet s'inscrivent dans le prolongement de celles rencontrées au sein de l'univers agricole et invitent à la comprendre comme un jardin singulier, « un jardin de viande » (roara ni noassi), où l'abondance et la variété des espèces sont également recherchées et maintenues pour des raisons esthétiques et sociales. L'ensemble de ces motifs nous incitent ainsi à qualifier les habitants de Tongoa comme des horticulteurs de la terre, mais aussi de la mer.

\section{BIBLIOGRAPHIE}

Artaud H. 2011 - La poḯtique des flots. Ouvrir, construire et refermer la mer dans le Banc d'Arguin, (Mauritanie). Thèse de doctorat en anthropologie, EHESS, sous la direction de Ph. Descola. Bambridge T. 2013 - Le foncier terrestre et marin en Polynésie française. L'étude de cas de Teahupoo. Revue des questions foncières (FAO) 2 (12) : 118-143.

Bambridge T. 2016 (Ed.) - The Rahui: Legal pluralism in Polynesia traditional management of resources and territories. Acton, ANU Press.

Bataille-Benguigui M-C. 1992 - Pêcheurs de terre, pêcheurs de mer. La mer dans la pensée tongienne. Études Rurales 127-128 : 55-73.

Bonnemaison J. 1981 - Voyage autour du territoire. Espace géographique 10 (4) : 249-262.

Bonnemaison J. 1996 - Gens de pirogue et gens de terre. Les fondements géographiques d'une identité, L'archipel du Vanuatu, Essai de géographie culturelle. Livre I: Gens de pirogue et gens de la terre. Paris, ORSTOM.

Burgos A. \& Dillais P. 2012 - Les femmes, les coquillages et la mangrove. Techniques \& Culture 59 : 326-337.

Calandra M. 2013 - Faire dessiner le terrain. La nature à « risques » et les jardins de subsistance de Tanna et Tongoa (Vanuatu). Techniques \& Culture $60: 182-201$.

Chapman L., Sharples P., Brogan D., Desurmont A., Beverly S. \& Sokini W. 2012 [2006] - Marine species identification manual for horizontal longline fishermen. Noumea, Secretariat of the Pacific Community.

Colding, J. \& Folcke C. 1997 - The relation between threatened species, their protection and taboos. Conservation Ecology 1 (1) : 1-6.

Cole-Dai J., Mosley-Thompson E. \& Thompson L.G. 1997 - Annually resolved southern hemisphere volcanic history from two Antarctic ice cores. Journal of Geophysical Research 102 (D14) : $16761-16771$.

Descola Ph. 2005 - Par-delà nature et culture. Paris, Gallimard. (Bibliothèque des Sciences humaines).

Dekker R.W., Fuller R.A. \& Baker G.C. 2000 - Megapodes. Statuts Survey and Action Plan 2000-2004, IUCN. 
Di Piazza A. \& Pearthree E. 2001 - L'art d'être pirogues de voyage en Océanie insulaire. Journal de la Société des Océanistes 112 : 61-72.

Dumas P., Jimenez H., Léopold M., Petro G. \& Jimmy R. 2010 - Effectiveness of village-based marine reserves on reef invertebrates in Emau, Vanuatu. Environmental Conservation 37 (3): 364-372.

Eissen J-P., Monzier M. \& Robin C. 1994 - Kuwae ( $\approx 1425$ A.D): the forgotten caldera. Journal of Volcanology and Geothermal Research 59 : 207-218.

Gibson J. 1979 - The ecological approach to visual perception. Boston, Houghton Mifflin.

Guiot H. 2003 - Les pirogues, reflets de la Polynésie. Dossier de la Société des Océanistes, Société des Océanistes, Paris.

Hickey F. 2007 - Gestion traditionnelle des ressources marines à Vanuatu : reconnaître, appuyer et renforcer les systèmes autochtones de gestion des ressources. Bulletin de la CPS 20 :11-23.

Hviding E. 1988 - Marine tenure and resource development in Marovo Lagoon, Solomon Islands: Traditional knowledge, use and management of marine resources, with implications for contemporary development, FFA Reports 88/35. Honiara, South Pacific Forum Fisheries Agency.

Hviding E. 1996 - Guardians of Marovo Lagoon: Practice, Place, and Politics in Maritime Melanesia. University of Hawai'i Press.

Hviding E. 2006 - Connaître et gérer la biodiversité dans les îles du Pacifique : problèmes posés par la préservation du lagon de Marovo. Revue internationale des sciences sociales 187 (1) : 73-90.

Ingold T. 2000 - The perception of the environment. Essays in livehood, dwelling and skill. London \& New York, Routledge.

Johannes R.E. \& Hickey F. 2004 - Evolution of village-based marine resource management in Vanuatu between 1993 and 2001. Paris, UNESCO. (Coastal region and small island papers ; 15).

Johannes R.E. \& Hviding E. 2002 - Le savoir traditionnel des pêcheurs du lagon de Marovo (îles Salomon) concernant le comportement grégaire des poissons. Bulletin de la CPS 12 :24-31.

Johannes R.E. 1981 - Words of the Lagoon: Fishing and Marine Lore in the Palau District of Micronesia. Berkeley, Los Angeles, London, University of California Press.

Leblic I. 2008 - Vivre de la mer, vivre avec la terre... en pays kanak. Savoirs et techniques des pêcheurs kanak du sud de la Nouvelle-Calédonie. Paris, Société des Océanistes.

Léopold M., Beckensteiner J., Kalvatara J., Raubani J., Carillon S. 2013 - Community-based management of near-shore fisheries in Vanuatu: What works? Marine Policy 42 (1) : 167-176.

McClanahan T.H., Glaesel H., Rubens J. \& Kiambo R. 1997 - The effects of traditional fisherie management on fisheries yields and the coral-reef ecosystems of southern Kenya. Environmental Conservation $24: 105-120$.

Merleau-Ponty M. 1945 - La Phénoménologie de la perception. Paris, Gallimard.

Peterson N. \& Rigby B. (Ed.) 2014 [1988] - Customary marine tenure in Australia. Sydney, Sydney University Press.

Roué M. 2006 - Introduction : entre cultures et natures. Revue internationale des sciences sociales 187 (1) : 11-18.

Ruddle K. \& Johannes R.E. (Ed.) 1990 - Traditional marine resource management systems in the Pacific Basin: An Anthology, Jakarta, UNESCO-ROSTSEA. 
Teulières-Preston M.H. 2000 - Le droit maritime kanak et ses transformations In : Bensa A. \& Leblic I. (Ed.) En pays kanak. Ethnologie, linguistique, histoire, archéologie en Nouvelle-Calédonie. Paris, Éditions de la Maison des sciences de l'homme : 129-146.

Vienne B. 1984 - Gens de Motlav. Idéologie et pratique sociale en Mélanésie. Paris, Société des Océanistes. (Publications de la Société des Océanistes ; 42).

\section{NOTES}

1. À Tongoa, deux langues coexistent: le nakanamanga (parlé par les habitants de Kurumampe) et le namakura.

2. Entre 2009 et 2015, des recherches ethnographiques pour le mémoire de master 1 et 2, puis pour le doctorat ont été conduites. Au total, 19 mois de terrain ont été entrepris dans le pays.

3. Cette île est à présent la plus grande du groupement central appelé Shepherd, mais aussi la plus densément peuplée de la région, puisqu'y vivent 2000 personnes, soit $48 \mathrm{hab} / \mathrm{km}^{2}$.

4. Cette transmission par l'oralité du système toponymique maritime est semblable à celle concernant le territoire terrestre.

5. Les parcelles cultivées sur l'île sont également nommées et leur toponymie permet aux horticulteurs de s'orienter sur l'île.

6. Il existe une expression synonyme également employée pour évoquer le large, il s'agit de natimboa qui pourrait être traduite par la phrase suivante : « là où l'on coule lorsque l'on n'a plus pied».

7. Bernard Vienne observe également ceci aux îles Banks (au nord du Vanuatu) et nomme cet espace la «brousse maritime» (1984:128).

8. Traduit du nakanamanga " roara ni noassi ».

9. Une première étude fut menée entre 1993 et 2001 par l'halieute-anthropologue Robert Edward Johannes et le biologiste Francis Hickey pour évaluer l'impact biologique des mesures de gestion locales au Vanuatu au sein de vingt-et-un villages côtiers. Le renouvellement des stocks d'espèces récifales telles que le trocas, la bêche de mer, le poulpe, le burgau, etc. fut ainsi étudié (Johannes \& Hickey 2004). Quelques années plus tard, des biologistes de l'IRD de Nouvelle-Calédonie ont de nouveau examiné l'efficacité de ce système de gestion des ressources. Ils ont conclu que si celuici était efficace dans le renouvellement des espèces sédentaires (coquillages et crustacés), il l'était bien moins pour les espèces migrantes (poissons). Les chercheurs ont suggéré aux chefs de la région de bloquer simultanément leurs parcelles afin d'étendre la zone de tapu et ainsi maximiser son incidence (Dumas et al. 2010, Léopold et al. 2013).

10. Citations issues du site internet: https://mol.gov.vu/index.php/en/acts-and-laws/232-thenew-land-laws-2014, page visitée le 05/10/2016.

11. La littérature concernant les restrictions coutumières est conséquente. On se rapportera notamment aux travaux de Ruddle \& Johannes 1990, Hviding 1996, 2006, Colding \& Folke 1997, Hickey 2007, McClanahan et al. 1997, Johannes \& Hviding 2002, Johannes \& Hickey 2004, Peterson \& Rigsby (Ed.) 2014 [1988], Bambridge 2016.

12. Le farea est un bâtiment en matériaux locaux d'une taille imposante (30 mètres de long sur 5 mètres de large). Il est le point focal de la vie collective du village.

13. En bislama cette expression suggère l'intelligence d'une personne. En français, on pourrait la traduire par : «ils ont de la réflexion » ou « ils ont des idées ».

14. Philippe Descola la définit comme «[...] une gamme de propriétés reconnues par tous les humains et recouvrant en partie ce que nous appelons d'ordinaire l'esprit, l'âme ou la conscience - intentionnalité, subjectivité, réflexivité, affects, aptitude à signifier ou à rêver. On peut aussi y inclure les principes immatériels supposés causer l'animation, tels le souffle ou l'énergie vitale, 
en même temps que les notions plus abstraites encore comme l'idée que je partage avec autrui une même essence, un même principe d'action ou une même origine. » (2005 : 168-169)

15. Au sens de Philippe Descola, c'est-à-dire: «[...] l'ensemble des expressions visibles et tangibles que prennent les dispositions propres à une entité quelconque lorsque celles-ci sont réputées résulter des caractéristiques morphologiques et physiologiques intrinsèques à cette entité » (2005: 169),

16. Au cours du terrain, nous avons observé qu'au-delà des trois premiers jours de deuil, les membres de la famille se lavent quotidiennement et se changent, ou alors se lavent et remettent les habits qu'ils portaient le jour du décès.

17. À l'entrée des maisons sont souvent accrochés des morceaux de coraux, car l'eau salée incrustée dans ces derniers, disent les Man-Tongoa, éloigne les mauvais esprits et protège des mauvais sorts.

18. Cette plante est utilisée pour marquer les interdits et accompagne les événements coutumiers importants, notamment lors de l'ordination des chefs ou la venue d'un homme important au village.

19. Lupalea est un village voisin de Kurumampe, voir la figure 2 .

20. Propos recueillis auprès de Tipea, le 05/06/2013 à Lupalea.

21. À titre d'exemple, lorsque souffle le vent du sud appelé siwo efate, les pêcheurs ne se rendent plus en mer, les vagues pouvant être dangereuses. Quand souffle le vent du nord-ouest one elao, il est dit que celui-ci persiste durant huit jours. Par conséquent, seule la façade est de Tongoa est navigable, car à l'abri du vent. C'est également one elao qui fait résonner sur l'île le bruit des vagues déferlant sur les rochers.

22. Ces relevés ont été réalisés à partir de l'ouvrage de Lindsay Chapman et al. (2012). Il est fort probable que certaines espèces aient échappé à notre recensement.

23. Fin août 2015, il y en avait quatre à Tongoa. Leurs propriétaires louent leurs services pour la pêche en haute mer, pour déposer les pêcheurs sur des îles voisines, notamment au sud d'Epi, située à une heure de navigation.

24. À notre connaissance, cette espèce ne fait l'objet d'aucun nasikoro. De nos jours, seuls des quotas sont imposés par le chef du village.

25. En 2013, le chef a limité la chasse à quarante oiseaux par personne. Cette année-là, dix-huit hommes y sont allés durant trois jours et ont ramené au village sept cent vingt pétrels, les bénéfices des ventes ont été reversés à l'Église presbytérienne.

26. Cette activité annuelle est la plus rentable de toutes celles pratiquées dans l'environnement marin. Les pétrels sont vendus au marché de Tongoa et sont parfois expédiés à Efate pour être également proposés sur les différents étals de l'île, mais à des prix bien plus onéreux.

27. Ariadna Burgos \& Peyo Dillais (2012 : 328) décrivent des associations écologiques similaires dans les pratiques de collectes des coquillages entreprises par les femmes de l'île de Siberut en Indonésie.

28. L'auteur fait là référence aux travaux du philosophe Maurice Merleau-Ponty (1945) sur la phénoménologie, et à ceux du psychologue américain James Gibson (1979 : 254).

29. À l'approche de Noël, les habitants disent : « kali pawa », « le bâton à fouir nous a épuisés », car toutes les semences d'ignames ont été mises en terre. Une période d'intense labeur dans les jardins vient là de prendre fin.

30. En 2015, la flottille du village comptait six pirogues (dont une en construction) pour une vingtaine de pêcheurs. 


\section{RÉSUMÉS}

À Tongoa, petite île située dans le groupement central des îles Shepherd au Vanuatu, la mer est pensée comme le prolongement de l'île. Les hommes possèdent des segments littoraux (natasi) et des parcelles de terre (roara) dont les ressources assurent l'alimentation quotidienne des familles. L'enquête ethnographique conduite dans le village de Kurumampe révèle que les parcelles de mer sont gérées et pensées comme des jardins. Les habitants sont autant des horticulteurs de la terre que de la mer. Cet article propose d'examiner ces différentes modalités d'usages et d'appréhension de l'environnement marin. Pour ce faire, le système foncier et la toponymie maritime, les différentes représentations de cet espace et les pratiques qui lui sont associées sont décrites et analysées.

On Tongoa Island, a small island located in the central Shepherd's archipelago in Vanuatu, the sea is considered as an extension of the land. Men own coastal plots (natasi) and allotments on the land (roara), on which they rely to feed their families. Our ethnographic research in Kurumampe village shows that sea plots are managed and perceived like subsistence gardens. The locals are horticulturists of both the sea and their land. This article examines the various modalities of use and apprehension of Tongoa's oceanic environment, by detailing and analysing the maritime tenure and toponymy, the representations of the sea and the local technics related to the coastal space.

\section{INDEX}

Keywords : sea, territory, fishing, horticulture, Tongoa

Mots-clés : mer, territoire, pêche, horticulture, Tongoa

\section{AUTEUR}

\section{MAËLLE CALANDRA}

Post-doctorante EPHE - Labex Corail-PSL /CREDO (UMR 7308 AMU-CNRS-EHESS) 